\title{
Can Nano-Impacts Detect Single Enzyme Activity? Theoretical Considerations and an Experimental Study of Catalase Impacts
}

\author{
Enno Kätelhön ${ }^{a}$, Lior Sepunaru ${ }^{a}$, Arkady A. Karyakin ${ }^{b}$, Richard G. Compton ${ }^{a}$ \\ ${ }^{a}$ Department of Chemistry, Physical and Theoretical Chemistry Laboratory, Oxford University, South Parks \\ Road, Oxford, OX1 3QZ, United Kingdom \\ ${ }^{b}$ Chemistry Faculty, M.V. Lomonosov Moscow State University, 119991, Moscow, Russia
}

\begin{abstract}
Recent publications report the direct electrochemical detection of individual enzyme activity via collisions at microelectrodes, though 'back of an envelope' calculations challenge the applicability of the experimental method. In the light of these studies, we present a comprehensive combined theoretical and computational analysis of the electrode response to a proximate and freely-diffusing enzyme generating an electroactive product. We further present experimental data for the enzyme catalase and critically discuss to what extent observed current transients can be unambiguously attributed to single enzyme collisions comparing detection via electrolysis of enzyme reaction products with detection through direct electron transfer reaction mediated via the enzyme.
\end{abstract}

\section{Introduction}

The scope of electrochemistry has recently broadened significantly with the introduction of the "nanoimpacts' technique ${ }^{1-3}$. In this, nanoparticles suspended in solution, by virtue of their Brownian motion, impact on a microelectrode. If the latter is held at a suitable potential then the arrival of the particle may be signalled by a burst of current lasting for the period of time in which the particle is in electrochemical contact with the electrode. The current can be due to direct electron transfer to or from the nanoparticles as in the oxidation of silver nanoparticles ${ }^{4}$ or the reduction of indigo ${ }^{5}$ or magnetite ${ }^{6}$ nanoparticles. Alternatively, the nanoparticle can mediate electron transfer bringing about redox chemistry associated with solution phase species as in the oxidation of hydrazine mediated via platinum nanoparticles ${ }^{7}$. The approach has rapidly expanded to embrace targets including liposomes ${ }^{8 ; 9}$, bacteria $^{10 ; 11}$, viruses $^{12 ; 13}$, micelles ${ }^{14}$ and diverse 'soft' nanoparticles ${ }^{9 ; 15}$.

The success of the nano-impact approach stimulated the question as to whether the electrochemistry of single enzyme catalytic activity can be visible via this or related approaches? Work by Hoeben ${ }^{16}$ et al. had demonstrated the ability to detect currents from a small ensemble of hydrogenase on a gold nanoelectrode. With the use of low-pass filters with a cutoff frequency of $30 \mathrm{~Hz}$, they were able to detect a catalytic current signal of $22 \mathrm{fA}$ arising from about 25 enzymes. This work is an important landmark which demonstrates the use of state of the art electrochemical instrumentation for achieving the minimal possible detected current. Currently, single enzyme catalytic activity can be detected by spectroscopic methods ${ }^{17 ; 18}$, which requires manipulation and modification of the enzyme under investigation. The aspiration to try and detect single enzyme activity with electrochemical methods arises from the inherent properties of the electrochemical system which permits in situ detection, without any manipulation of 
the biomolecule examined. One approach which, at least at first sight, might be viable, is to follow a hint by Bard ${ }^{19}$ in a paper entitled 'Toward Single-Enzyme Molecule Electrochemistry', in which he suggests possible enzyme detection via amplification of the current from the electroactivity of a catalytically formed product. Related to this interest, recent studies explore apparent nano-impacts seen resulting from the laccase catalysed four electron reduction of oxygen ${ }^{20}$ and the horse radish peroxidase catalysed hydrogen peroxide reduction ${ }^{21}$ and attribute observations to single enzyme-electrode collisions coupled to possible direct electron transfer, a quite different mechanism and reaction to that envisaged in the product detection approach.

The following 'Back of the envelope' estimation based on the enzyme turnover number suggest that a simple model in which an impacting enzyme hits an electrode and catalyses the conversion of a substrate to a product may be inconsistent with the observed magnitude of the 'nano-impacts' if the latter result in the electrolysis of the products. Enzyme catalytic activity in an ensemble is characterized by the catalytic constant $\mathrm{k}_{\text {cat }}$, which is also referred to as the limiting constant or turnover number. It means that the reaction rate cannot be faster than the enzyme concentration multiplied by this catalytic (limiting) constant. The dimensions of the latter are reciprocal seconds $\left(\mathrm{s}^{-1}\right)$. Accordingly, to estimate the upper limit value of current generated by the single enzyme molecule the following equation is valid:

$$
I_{e l}=k_{c a t} e_{0} n
$$

where $e_{0}$, the electron charge, equals $1.602 \cdot 10^{-19} \mathrm{C}$ and $n$ is the number of electrons transferred per Faradaically per consumed substrate molecule. In the article ${ }^{20}$ current spikes of 3-4 pA are reported, which are attributed to the current of a single laccase molecule. This, considering 4 electrons per turnover, requires a catalytic constant greater than $10^{7} \mathrm{~s}^{-1}$. However, for laccase, the highest catalytic constant ever reported is $500 \mathrm{~s}^{-1} .{ }^{22}$ Hence the reported current attributed to a single laccase molecule is about $10^{5}$ times higher than the limiting theoretical value. It is noted that literature ${ }^{17}$ describes fluctuations in the activity of individual enzymes. The observed deviations from the ensemble mean were, however, of far smaller magnitude. Moreover, the literature suggests that on gold electrodes the enzyme laccase from a similar source does not display any electrocatalytic activity. ${ }^{23}$ The observation of a single impacting peroxidase molecule ${ }^{21}$ is less contradictory to literature, however, the reported catalytic constant of $10^{6}$ $\mathrm{s}^{-1}$ far exceeds the expectation as for native peroxidase the turnover number in direct bioelectrocatalysis on gold electrodes was measured ${ }^{24}$ to be only $1 \mathrm{~s}^{-1}$. Even for recombinant peroxidases, which in contrast to the native enzyme do not feature a hydrocarbon shield, the turnover numbers are $20-30 \mathrm{~s}^{-1}$, which is 100,000 times smaller than the value reported $\mathrm{in}^{21}$. An improvement in turnover number as suggested for laccase has never been observed in bioelectrocatalysis except in the report ${ }^{20}$ which is mentioned above.

The nano-impacts experiment, whilst at first sight is attractively simple, requires careful analysis especially in respect to the size and frequency of impacts as well as possible influences from the measurement's electronics. In the following we first present a theoretical/computational analysis of the nano-impact experiments as applied to collisions of a single enzyme with an electrode and assume that the former reacts with a target substrate according to Michaelis-Menten kinetics ${ }^{25 ; 26}$ and that the product of the enzyme reaction is detected electrochemically. We further present experiments relating to the enzyme catalase and its reaction with $\mathrm{H}_{2} \mathrm{O}_{2}$ and critically assess whether the observed current spikes can be realistically attributed to single enzyme events following the assumed mechanism, whilst considering alternatives, and in particular the possibility of direct electron transfer mediated via the impacting enzyme. 


$$
\begin{gathered}
E+S \stackrel{\text { enzymatic }}{\stackrel{\text { diffusive }}{\longrightarrow}} \text { PS } \stackrel{\text { enzymatic }}{\longrightarrow} E+P \\
P \stackrel{\text { bulk }}{\longrightarrow} P_{\text {electrode }} \\
P \stackrel{\text { diffusive }}{\longrightarrow \text { Faradaic }} P_{\text {electrode }}^{-n_{e}}
\end{gathered}
$$

Figure 1: Scheme of the modelled reaction. Enzymes $E$ located in the bulk reach the electrode by virtue of their Brownian motion, where they catalytically turn substrate $S$ into product $P$. The product may then either be electrochemically detected at the electrode or diffuses into the bulk where it remains. For clarity different subscripts differentiate between product located in the bulk and in the electrode's proximity, as only the latter can be detected. It is however noted that in our model enzymes remain active at all time independent of their position.

\section{Theory}

In the following theoretical section, we discuss challenges and opportunities arising in the detection of individual enzyme activity through electrochemical methods. As the description of the corresponding experimental set-up renders a complex multi-physics problem, we subdivide our investigation into the analysis of elementary underlying processes that are each individually addressed, mathematically modelled, and then combined to provide a coherent picture.

We first introduce the general theoretical model before we address the diffusive mass transport of the enzymes' products. Herein, we particularly focus on their electrochemical detection and reveal its dependence on the point of release relative to the electrode. We subsequently examine the mass transport of the enzyme itself and assess different modelling approaches. Furthermore, we comment on the challenges arising from the use of bandwidth-limited analogue laboratory equipment in the experimental measurement procedure and discuss to which extend an analysis may be complicated or even inhibited. Last, we merge our results in the presentation of a comprehensive theoretical modelling approach.

\subsection{Theoretical model}

We consider the detection of a freely diffusing enzyme via electrochemical measurement of its product. Our model considers a solution comprising an excess amount of the enzyme's substrate and initially a negligible amount of enzyme product. We suppose that the enzyme kinetics follow the Michaelis-Menten model and steadily transform substrate into product independent of the enzyme position. Figure 1 depicts the scheme of detection: Enzymes $E$ diffusively travel through solution, where they transform substrate $S$ into product $P$ along their path. Product molecules then either diffuse towards the electrode where they are detected or into bulk where they remain. All constants related to Michaelis-Menten kinetics are further assumed to exhibit only small variations over time and in different enzymes of the same species, in particular the enzyme's static and dynamic disorder in turnover number is neglected.* All mass transport of the enzyme, the substrate, and the product is considered to be solely diffusional, i.e. there is no convection and diffusing species do not experience any electric fields. The enzyme is however affected by near-wall hindered diffusion, which retards its diffusion perpendicular to planar solid boundaries in the proximity thereof. As discussed below, enzymes are herein modelled as rigid spherical objects, which enables modelling hindered diffusion on the basis of well-established theory.

The chosen geometry is a nano-impact experiment at a macro- or recessed micro disk electrode allowing simplification to one dimension: On one side, the space is confined by a solid boundary that represents the electrode while the opposite boundary is assumed to be in sufficient distance to not influence processes at 


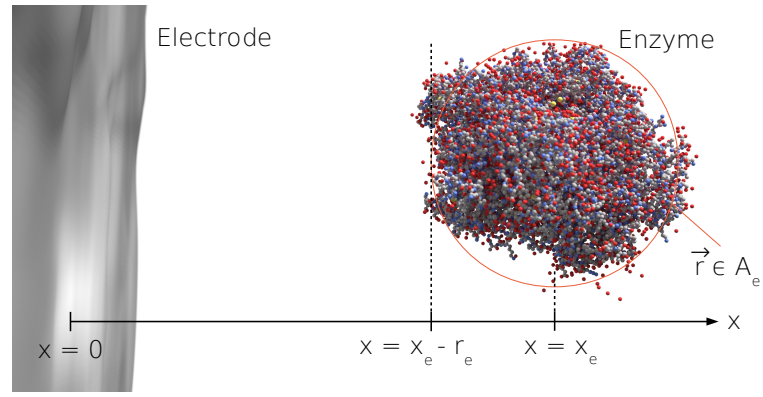

Figure 2: Illustration of the modelled geometry.

\begin{tabular}{|c|c|c|c|c|}
\hline Parameter & Material & Symbol & Value & Unit \\
\hline Enzyme radius & Bovine liver catalase $^{27}$ & $r_{e}$ & 5.22 & $\mathrm{~nm}$ \\
\hline Enzyme maximum turnover number ${ }^{\ddagger}$ & Bovine liver catalase ${ }^{28}$ & $k_{\text {cat }}$ & $7.24 \cdot 10^{5}$ & $\mathrm{~s}^{-1}$ \\
\hline Enzyme diffusion coefficient ${ }^{\S}$ & Bovine liver catalase & $D_{e}$ & $4.18 \cdot 10^{-11}$ & $\mathrm{~m}^{2} \mathrm{~s}^{-1}$ \\
\hline Michaelis-Menten constant & Bovine liver catalase $^{28}$ & $K_{M}$ & 93 & $\mathrm{mM}$ \\
\hline Substrate diffusion coefficient & Hydrogen peroxide ${ }^{29}$ & $D_{s}$ & $1.71 \cdot 10^{-9}$ & $\mathrm{~m}^{2} \mathrm{~s}^{-1}$ \\
\hline Product diffusion coefficient & Oxygen $^{30}$ & $D_{p}$ & $1.76 \cdot 10^{-9}$ & $\mathrm{~m}^{2} \mathrm{~s}^{-1}$ \\
\hline Charge transfer in Faradaic reaction & Oxygen & $q_{0}=-n_{e} e_{0}$ & $2 e_{0}$ & $\mathrm{C}$ \\
\hline
\end{tabular}

Table 1: Parameters used in the theoretical modelling.

the electrode, see Figure 2. The electrode boundary is reflective to the enzyme and the substrate, which neither react nor adsorb at the surface, but immediately consumes the product. We hence delineate an electrochemical reaction of the product at an electrode featuring a sufficiently high over- or underpotential to drive the reaction.

In this work, we model impact experiments with the enzyme catalase. Herein, hydrogen peroxide is catalysed $^{\dagger}$ to oxygen, which is electrochemically detected at the electrode:

$$
\begin{aligned}
2 \mathrm{H}_{2} \mathrm{O}_{2} \stackrel{\text { enzymatic }}{\longrightarrow} & 2 \mathrm{H}_{2} \mathrm{O}+\mathrm{O}_{2} \\
2 \mathrm{H}^{+}+\mathrm{O}_{2}+2 e \stackrel{\text { Faradaic }}{\longrightarrow} & \mathrm{H}_{2} \mathrm{O}_{2}
\end{aligned}
$$

All parameters employed are listed in Table 1.

\section{$2.2 \quad$ Electrochemical detection of the enzyme product}

During the impact, the presence of the enzyme in the proximity of the electrode surface is solely monitored via the electrochemical detection of the product arising from its reaction with a solution phase substrate $S$. Product molecules are generated at the diffusing enzyme and stochastically reach the electrode by virtue of their Brownian motion. The detection of the enzyme is hence indirect and depends on the enzyme's diffusive pathway relative to the electrode surface. While theory on the movement of the enzyme is

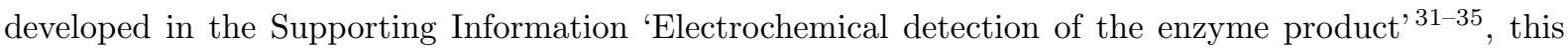
section compiles the key findings on the mass transport of individual product molecules: We establish an analytical expression for the temporal distribution of detection events and determine the probability of a product molecule to be detected. In combination with knowledge on the enzymes mass transport and the signal transfer characteristics of the measurement equipment, this will enable us below to predict the potentiostat response to an impacting enzyme.

By solving the one-dimensional diffusion equation, we determine the average current $I_{0}$ that results from a product molecule being released at the enzyme position $x_{e}$ at the time $t_{0}$ : 


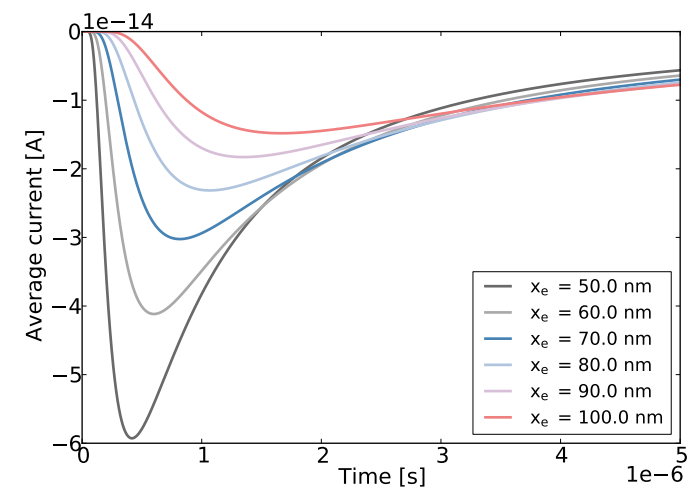

Figure 3: Average electrode current modelled for a single product molecule released at the time $t=0$ at the position $x_{e}$. Due to the discrete nature of the product molecules the average current can be interpreted as a probability density for a reaction at the time $t$. The diffusion coefficient of the product is set to $D_{p}=10^{-9} \mathrm{~m}^{2} \mathrm{~s}^{-1}$.

$$
I_{0}\left(t, t_{0}, x_{e}\right)=\frac{q_{0} x_{e}}{\sqrt{4 \pi D_{p}} \cdot\left(t-t_{0}\right)^{3 / 2}} \exp \left(-\frac{x_{e}^{2}}{4 D_{p}\left(t-t_{0}\right)}\right)
$$

where $D_{p}$ is the diffusion coefficient of the product and $q_{0}$ the charge transferred at the electrode. Herein it is noted that the transferred charge features a discrete nature and the provided equation can hence be interpreted as a temporal probability density of the charge transfer. Figure 3 depicts this expression for various distances $x_{e}$ of the release position from the electrode.

Equation 3 further enables us to determine the probability of a product molecule reaching the electrode at all. To this end, we integrate the current (3) caused by a single product molecules being released, in the time interval $\left[t_{0}, \infty\right)$ and find:

$$
\int_{t_{0}}^{\infty} d t I_{0}\left(t, t_{0}, x_{e}\right)=q_{0}=-n_{e} e_{0} \text { for } x_{e} \in \mathbb{R}^{+}
$$

The charge transferred per product molecule in infinite time equals the maximum charge a product molecule can transfer. Every single product molecule hence eventually reaches the electrode independent of its release positions at some point in time.

\subsection{Mass transport of enzymes}

Various approaches can be readily pursued to model enzyme mass transport. Most prominently mass transport equations along the lines of diffusion equation are solved via finite-element or finite-difference methods. A concentration profile or in the case of a single enzyme a probability density of the enzyme position as a function of time is then determined, as the discrete nature of a diffusing object is not considered. While this approach conveniently enables the calculation and modelling of the substrate and product diffusion, where we consider a large number of molecules and individual diffusive paths are not interest, other methods must be employed for the modelling of the enzymes' Brownian motion. Since the given problem however requires knowledge of the stochasticity of the enzymes path, solving diffusion equation bears the disadvantage that the needed path is not directly accessible from the result. Stochastic modelling via random walks ${ }^{36}$ hence renders a more suitable approach as it provides stochastic enzyme positions as a function of time, though at the expense of a time-discrete solution and the negligence of features beyond the bandwidth thereof.

Due to the enzymes' size, Brownian motion is affected by the effect of near-wall hindered diffusion: 
Hydrodynamic effects in the solvent leads to a hindrance of the enzymes diffusion near the electrode surface, which manifests in an increasingly reduced and anisotropic diffusion coefficient in the proximity of the surface. The impact of this effect on the Brownian motion of the enzyme is herein dramatic: While at large distances perpendicular diffusion remains unaltered, in the immediate proximity to the surface perpendicular diffusion is entirely suppressed and no Brownian motion occurs in this direction. Previous theoretical analysis of the phenomenon lead to an expression for the diffusion coefficient $D_{e, \perp}$ of a spherical particle diffusing perpendicularly to a flat boundary ${ }^{37 ; 38}$ :

$$
D_{e, \perp}\left(x_{e}\right)=\frac{6\left(x_{e}-r_{e}\right)^{2}+2 r_{e}\left(x_{e}-r_{e}\right)}{6\left(x_{e}-r_{e}\right)^{2}+9 r_{e}\left(x_{e}-r_{e}\right)+2 r_{e}^{2}} D_{e}
$$

where $x_{e}-r_{e}$ is the distance between the surface and the point on the particle surface that is closest to the surface. This expression can directly be used for the construction of a random walk algorithm as shown in recent literature that discussed various approaches for the implementation of such anisotropic diffusion into random walks. Most commonly random walks are approximated as ${ }^{39}$ :

$$
x_{e, k}=x_{e, k-1} \pm \sqrt{2 D_{e, \perp}\left(x_{e, k}\right) \cdot \Delta t}
$$

where $x_{k}$ is the position of the enzyme at the time $t_{k}$ and \pm is evaluated at a probability of $1 / 2$. It is however noted that this modelling leads to a drift of the random walker towards the electrode and hence yields an overestimation of particles' time of residence in the proximity of the boundary. This inaccuracy can however be compensated for by the inclusion of an additional position-dependant drift term as discussed by Kätelhön et al. ${ }^{40}$. In many cases Equation (6) may nonetheless provide an acceptable model for anisotropic diffusion. Particularly with respect to the here-investigated problem, the accuracy of this model fully satisfactory as we will see below.

\subsection{Complications arising from analogue measurement circuitry}

A major challenge in the signal analysis in nano-impact experiments lies in the understanding of the influence of employed measurement circuitry. It is by no means given that experimentally measured currents as recorded by a potentiostat represent the charge transfer at the electrode-electrolyte interface. In contrast rather a fingerprint of the actual interface process is depicted as the signal is often drastically obscured by the measurement set-up. If no digital filters are employed by the potentiostat and its control software, there are three elements of the measurement equipment that mainly cause these alterations - namely analogue filters set before the analogue-digital converter, the analogue-digital converter itself, and inevitable parasitic capacitances that occur at cables, connectors, plugs, and so on. It was shown recently ${ }^{41}$ that among these the filter employed dominates the measurement and the influence of the analogue-digital converter and parasitic capacitances can usually be neglected.

Two studies investigated the influence of analogue filters on measured nano-impact spikes and demonstrated that measured spike heights and -widths can be altered by an order or even orders of magnitude ${ }^{42}$, or may be entirely masked by the measurement circuitry ${ }^{41}$. In the first case features of the original spike shape may still be retained from the data, in the latter, however, all information on the shape is lost. Unless the exact specifications of the measurement set-up are known, it is therefore crucial for experimentalists to exclude spikes features such as spike heights or -widths from any quantitative analysis as the measured values may be entirely obscured. Otherwise the filter response must be known to ensure that information of interest is either conserved or altered in a way that enables a later recovery of the desired original data. It is though noted that this limitation only applies to alternating-current signals. If for instance potential steps are recorded and (only) if the current after the step remains at a constant value, the step height may be used in a quantitative analysis though the step transient may be masked 
by the filter.

While the analysis of data relating to spike shape features may be complicated by analogue circuitry, the overall charge transferred per spike is typically conserved in commercially-available potentiostats. ${ }^{41}$ Various studies compared the size distributions of particle populations as obtained from the charge transfer in nano-impact experiments and electron microscopy measurements, where excellent agreement was found. This can be understood via the characteristics of the employed filters. Many of the commonly used analogue filters resemble Butterworth- or Bessel-type filters, which are infinite impulse response (IIR) filters and conserve the integral of an impulse. If an impulse that features a bandwidth beyond the bandwidth of the filter is measured, the filter response will be an entirely different pulse though the integral value is conserved.

If the exact transfer function $H(s)$ of the filter is known, continuous-time input- and output signals, $x(t)$ and $y(t)$ relate as follows ${ }^{43}$ :

$$
\frac{\mathcal{L}(y(t))}{\mathcal{L}(x(t))}=H(s)
$$

where $\mathcal{L}$ depicts the Laplace transformation and $s \in \mathbb{C}$ the Laplace frequency parameter. In the case of time-discrete data that prohibits the use of the Laplace transform, the filter response can instead be modelled via the z-transform as discussed in previous publications and text books ${ }^{44}$. The transfer function is then conformally mapped into z-space and the z-transforms of the time-discrete input- and output signals are evaluated.

\subsection{Modelling approach}

We can now merge the results of previous sections to design a simulation of the potentiostat's current response to an impacting enzyme. Using the time-discrete enzyme path $x_{k}\left(t_{k}\right)$ modelled via Equation (6), we can determine the spatotemporal distribution of product molecules being released. According to the reaction equation (2) of catalase, at each position $x_{k}$ at the time $t_{k}$ the enzyme releases $k_{c a t} \cdot \Delta t$ product molecules, where $\Delta t=t_{k+1}-t_{k}$ is the temporal step width of the random walk. On the basis of Equation (3), we can then construct the Faradaic current $I_{e l}$ at the electrode as the superposition of the currents caused by each individual molecule:

$$
I_{e l}(t)=v \Delta t \sum_{k} I_{0}\left(t, t_{k}, x_{e, k}\right)
$$

The current $I_{\text {exp }}$ that is measured by the potentiostat can then be found through the inclusion of the filter via Equation (7):

$$
I_{\text {exp }}(t)=\mathcal{L}^{-1}\left(\mathcal{L}\left(x_{e}(t)\right) \cdot H(s)\right)
$$

where $\mathcal{L}^{-1}$ is the inverse Laplace transform. ${ }^{1}$

\section{Computational methods}

We develop code to simulate the enzyme path $x_{e}$ and the electrode current $I_{e l}$. To this end, the enzyme path is modelled via random walks on the basis of Equation (5) and (6), while we impose a minimum distance $^{39}$ of $h_{\min }=0.1 \mathrm{~nm}$ between the random walker's edge and the electrode on the enzyme's

\footnotetext{
${ }^{1}$ It is noted that Equation (8) is time-continuous even though the enzyme path and the time distribution of its product release are not. The Laplace transform can therefore be employed and modelling the filter via the z-transform is not required.
} 


\begin{tabular}{lllll} 
& Parameter & Symbol & Value & Unit \\
\hline Random walk: & Initial position & $x_{e}(t=0)$ & 100 & $\mathrm{~nm}$ \\
& Minimum distance to electrode & $h_{\min }$ & 0.1 & $\mathrm{~nm}$ \\
& Random walk temporal step width & $\Delta t$ & $10^{-7}$ & $\mathrm{~s}$ \\
Electrode current: & Threshold in the calculation of $I_{0}$ & $\epsilon$ & 1 & $\%$ \\
Potentiostat modelling: & Filter cutoff frequency & $f_{c}$ & 100 and 4000 & $\mathrm{~Hz}$
\end{tabular}

Table 2: Additional simulation-specific parameters extending Table 1.

Brownian motion. The obtained positions are used to determine $I_{e l}$ as shown in Equation (8). Herein, we significantly limit the computational effort through the introduction of a threshold factor $\epsilon$ : Whenever an individual current contribution $I_{0}\left(t, t_{k}, x_{e, k}\right)$ in Equation (8) for a given enzyme position $x_{k}$ drops below the value $\epsilon \cdot \max \left(I_{0}\left(t, t_{k}, x_{e, k}\right)\right)$, its further contribution to $I_{e l}$ is neglected. The simulation hence underestimates the observed current in return of a better computational performance. Our model further includes the impact of the potentiostat's analogue circuitry along the lines of Equation (9). To this end, we include a digital first-order Butterworth low-pass filter operated at a cutoff frequency $f_{c}$ of $100 \mathrm{~Hz}$ and $4 \mathrm{kHz}$.

The random walk and the calculation of $I_{e l}$ are implemented in $C++$ and employ the pseudo random number generator Mersenne Twister ${ }^{45}$ in its 2002 revision via the GNU Scientific Library (GSL). The inclusion of the Butterworth filter as a digital filter is put into effect via the SciPy package in Python. All simulation parameters extending the physiochemical parameters provided in Table 1 are given in Table 2 .

\section{Experimental methods}

Sodium chloride, monopotassium phosphate $\left(\mathrm{KH}_{2} \mathrm{PO}_{4}, \&\right.$ i 99$)$, dipotassium hydrogenphosphate $\left(\mathrm{K}_{2} \mathrm{HPO}_{4}\right.$, ¿ 98\%) and the enzyme catalase from bovine liver were acquired from Sigma (St. Louis, MO, USA). All solutions were made on the basis of ultra pure water (Millipore Billerica, MA, USA) featuring a resistivity of $18.2 \mathrm{M} \Omega \mathrm{cm}$ at $298 \mathrm{~K}$, and comprise $100 \mathrm{mM} \mathrm{K}_{2} \mathrm{HPO}_{4} / \mathrm{K}_{2} \mathrm{H}_{2} \mathrm{PO}_{4}$ phosphate buffer as well as 140 $\mathrm{mM} \mathrm{NaCl}$. The phosphate buffer was adjusted to $\mathrm{pH}$ 7, which was confirmed through a Hanna pH 213 pH-meter. Prior to use, solutions were deoxygenated by bubbling oxygen-free nitrogen (BOC, Guildford, UK) for at least 15 minutes.

Electrochemical measurements were performed using a $4.9 \mu \mathrm{m}$ carbon microelectrode as the working electrode. Prior to use, the electrode was polished with MicroPolish II alumina suspensions (Buehler, Lake Bluff, IL, USA) comprising decreasing particle sizes of 3.0, 0.1, and $0.01 \mathrm{~mm}$ and washed thoroughly with water. Experiments were conducted at $295 \mathrm{~K}$ within a Faraday cage where measurements were made versus a saturated calomel reference electrode (SCE) and a graphite rod counter electrode. A home-made potentiostat $^{46}$ featuring a $4 \mathrm{KHz}$ preamplifier (type $L C A-4 K-1 G$, Laser Components GmbH, Olching, Germany) was employed in all experiments and data was used without any further filtering.

\section{Results and discussion}

The following section is structured into three parts. First we present simulation results for an enzyme impacting from a solution that contains equally-distributed substrate, which enables us to draw general conclusions regarding the feasibility of single enzyme detection via this type of impact approach. In the light of these results, we subsequently interpret experimental results of the enzyme catalase. 
a)

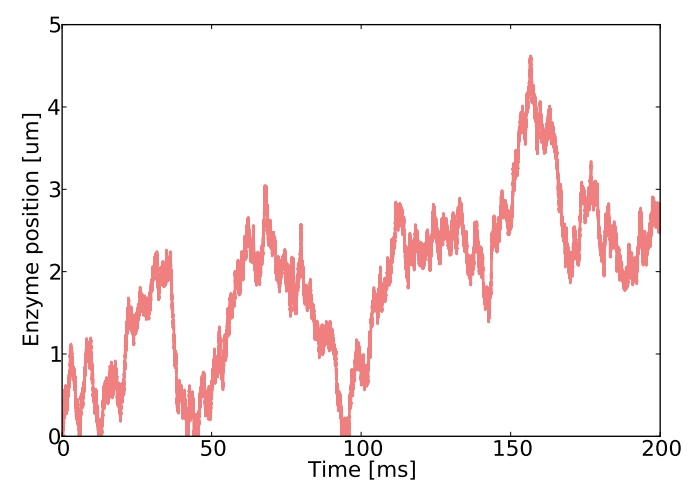

b)

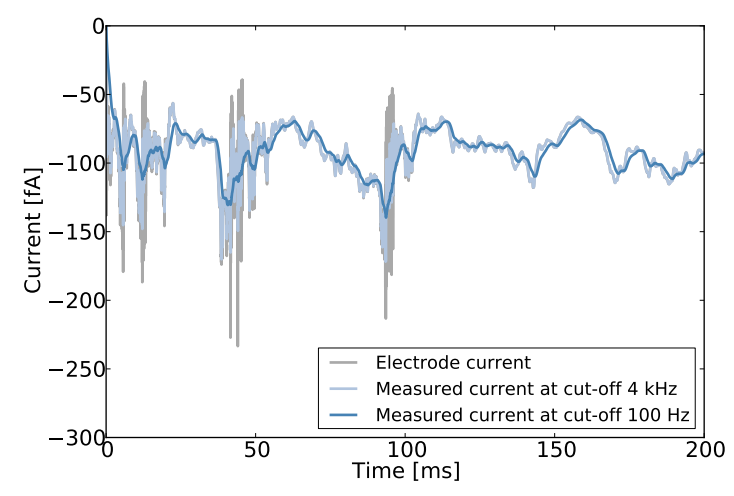

Figure 4: Simulation results for catalase: a) Diffusive path of the enzyme in the proximity of the electrode at $x=0$. b) Modelled electrode current resulting from the enzyme path depicted in a). The light grey graph presents the current at the electrode surface, whereas the two blue graphs show currents actually measured by simulated potentiostats using two different filters.

\subsection{Simulation}

We model an exemplary nano-impact event of a single catalase molecule. The enzyme is virtually placed in a solution that comprises no product molecules but an excess amount of substrate, hence allowing enzymatic activity at the maximum turnover number $k_{\text {cat }}$. The catalase molecule is further initially released at a distance of $x_{e, 0}=100 \mathrm{~nm}$ from the electrode to diffuse freely though affected by near-wall hindered diffusion. On the basis of the above random walk model (6) and the analytical expression (8) for the diffusion of product towards the electrode, we model a possible diffusion path of an enzyme as well as the expected electrode response to product molecules generated along the same path. Potentiostat characteristics as subsequently exemplified along the lines of Equation (9) by including a digital Butterworth filter operated at a cut-off frequency $f_{c} 100 \mathrm{~Hz}$ and $4 \mathrm{kHz}$. Results for the enzyme position and the electrode current in comparison to the current as measured by the potentiostat are shown in Figure 4 a) and b), respectively.

The simulated random path models a time interval of $200 \mathrm{~ms}$, during which the random walker approaches the electrode surface several times while remaining within a distance of $5 \mu \mathrm{m}$ throughout the experiment. This does however counter-intuitively not lead to 'spike'-like features being observed at each impact, but an almost constant current independent of the enzyme position if averaged over few tens of milliseconds. The finding can be understood considering the large difference in diffusion coefficient between the enzyme and its products, which amounts to about two orders of magnitude: The enzyme is almost stationary on the time scale of product diffusion, and since all product molecules 
eventually inevitably reach the electrode surface as demonstrated through Equation (4), the electrode current must tend towards a constant value if sampled at a low bandwidth. This effect is independent of the enzyme turnover number as more active enzymes or agglomerates equally would cause an average current independent of their position rather than spikes.

The simulated path is nonetheless represented in the electrode current in various ways: First, we notice a trend that to some extent the slope of both graphs appear to be linearly coupled. Whenever the enzyme moves towards the electrode, i.e Figure 4 a) exhibits a negative slope, the absolute electrode current increases, while it decreases as the enzyme diffuses towards the bulk. As the enzyme approaches the electrode, the rate of detection of the steadily generated product increases and vice versa. Second, we observe that the same effect leads to significantly greater noise when the enzyme is located in close proximity to the electrode, where the coupling between the enzyme position and the current triggered by the Faradaic reaction of its products increases. This finding can be explained via Equation (8), which provides the current response to a single product molecule as a function of time: As the distance $x_{e}$ between the point of release and the electrode tends towards zero, the resultant electrode current $I_{0}$ adopts the form of a $\delta$-function at the time $t_{0}$ of the product release, while the transition towards the $\delta$-function exhibits a strong dependency on $x_{e}$. In contrast to that, changes in $x_{e}$ at large distances only lead to small variations in the current response. The coupling between the enzyme movement and the electrode current is hence stronger at small distances, which we find reflected in the noise of the simulated electrode current.

The exploitation of the noise-distance dependence of $I_{e l}$ on $x_{e}$ for the reconstruction of the enzyme path is however obstructed as the required data is typically not experimentally accessible. As it can be seen in Figure $4 \mathrm{~b}$ ) the limited bandwidth of the potentiostat effectively masks the noise characteristics needed for analysis. Regions of the graph featuring greater variance are smoothed to an extent that they cannot be distinguished from other actually smooth regions. Even if higher-bandwidth potentiostats were employed, the analysis would nonetheless be experimentally complicated as numerous other noise sources may occur in similar frequency regimes. These may for instance include thermal capacity fluctuations at the electrode-electrolyte interface, intrinsic noise resulting from the discrete nature of Faradaic charge transfer at the electrode, thermal noise in analogue circuitry, and analogue-digital converters to just name a few.

\subsection{Experiments}

To complement our theoretical results, we experimentally investigate the feasibility of single-enzyme detection via electrochemical methods. To this end, we exemplarily demonstrate nano-impact experiments of the enzyme catalase, which presents an excellent model system for various reasons:

- The possibility of single enzyme detection either via product electrolysis or direct electron transfer can be explored. ${ }^{47 ; 48}$

- Catalase decomposes hydrogen peroxide into oxygen at a high turnover rate of $7.24 \cdot 10^{5} \mathrm{~s}^{-1} .^{28}$

- The rate of enzymatic hydrogen peroxide disproportionation obeys Michaelis-Menten kinetics up to high substrate concentrations of $0.2 \mathrm{M} .^{28}$

- Hydrogen peroxide serves as the single substrate for the enzyme, which enables experiments in a solution comprising only two electroactive substances, namely the enzyme's substrate and its product.

- Catalase remains active in the proximity of a carbon electrode. It was recently shown that a carbon electrode modified with catalase can perform a near four-electron reduction of oxygen at relativity 
low overpotentials. ${ }^{49}$

In our nano-impact experiments, we investigated a solution comprising $9 \mathrm{pM}$ catalase and $100 \mathrm{mM}$ $\mathrm{H}_{2} \mathrm{O}_{2}$ in a PBS buffer. A potential of $-1.0 \mathrm{~V}$ versus SCE, which is sufficiently negative to enable oxygen reduction at the electrode,${ }^{48}$ is applied at a carbon microelectrode. As higher overpotentials can cause reduction of hydrogen peroxide at the electrode surface, such are avoided to ensure that Faradaic current solely originate from the oxygen reaction. Figure 5 depicts typical experimental results found under these conditions. Numerous reductive spikes are observed featuring a varying nature with about half of the spikes exhibiting sharp, possibly bandwidth-limited shapes. Two typical spikes are amplified in Figure $5 \mathrm{~b}$ ) and c), where we find such sharp transient and a 'burst' of overlapping current events that cannot be resolved individually, respectively. It is further noted that spikes cannot be seen in absence of the enzyme (data not shown).

Direct comparison of Figure 5 with the simulation results for the one-dimensional model in Figure 4 immediately reveals drastic differences between theoretically predicted currents and experimental observations. While simulations suggest that independent of the turnover rate no spikes can be found in current measurements of both impacting individual enzymes as well as impacting enzyme clusters, experiments see a large number thereof. Theory based on Michaelis-Menten kinetics further expects a maximum current contribution of -116 fA per enzyme as determined analytically through $\left\langle I_{0}\right\rangle=e_{0} k_{c a t}$ on the basis of Michaelis-Menten kinetics. If experimental spikes were interpreted as Faradaic currents resulting from the reduction of the products of individual enzymes, experimental results would hence exceed theoretical expectations by three orders of magnitude! Though variations in the activity of individual enzymes have been reported, we consider this value to be unrealistically high as it would require huge ratios of $10^{3}$ between inactive and active periods to compensate for the difference.

If we took the view that the Michaelis-Menten model as parametrised in Table 1 was not applicable to enzymes at the electrode surface while such enzymes would nonetheless remain enzymatically active, other options for interpretation would arise. When comparing experimental results with the above hypothetically assumed diffusion-limited kinetics of an uniformly-accessible enzyme mounted on a surface, we find that experimental results are within the predicted limiting value of $0.75 \mathrm{nA}$ per enzyme and numerous spikes exceed $10 \%$ of this limit. Herein, it is however crucial to consider the measurement characteristics of potentiostat: While longer spikes such as the spike in Figure 5c) are likely not affected by bandwidth limitations, the actual spike heights of shorter spikes such as the spike depicted in Figure 5c) may be much higher and can therefore not be used for analysis unless the filter's impulse response is known. Either way, results suggest more a diffusion- than a kinetically controlled Michaelis-Menten type reaction. It is though noted that the analysis in the Supporting Information 'Enzyme activity in the diffusion limit' reveals that diffusion-controlled enzyme reactions require enzyme radii of only $0.7 \mathrm{~nm}$ to produce a steadystate current of $100 \mathrm{pA}$, which is much below the actual enzyme radius. This finding rather suggests that enzymes either undergo drastic changes near the electrode, if the observed spikes are to be attributed to products from single enzymes, or else other effects such as direct electron transfer are taking place.

We therefore adopt the view that measured spikes cannot be unambiguously attributed to impacts of individual enzymes via product detection and speculate that experimental observations have different origins. Facilitated by the presence of enzymes, nanobubbles could for instance be formed in solution and capacitively interact with the electrode surface. Another distinct possibility includes Faradaic currents enabled by enzymes temporarily acting as nanoelectrode in a diffusion-controlled or near diffusion-controlled reaction - so-called 'direct electrochemistry'. Moreover, the net charge transfer would imply a change in the products formed in the enzyme reaction. Nevertheless one could imagine a scenario in which the substrate binds to the impacting enzyme as in the Michaelis-Menten model followed by a rapid electron transfer leading possibly to the reduction of oxygen: 

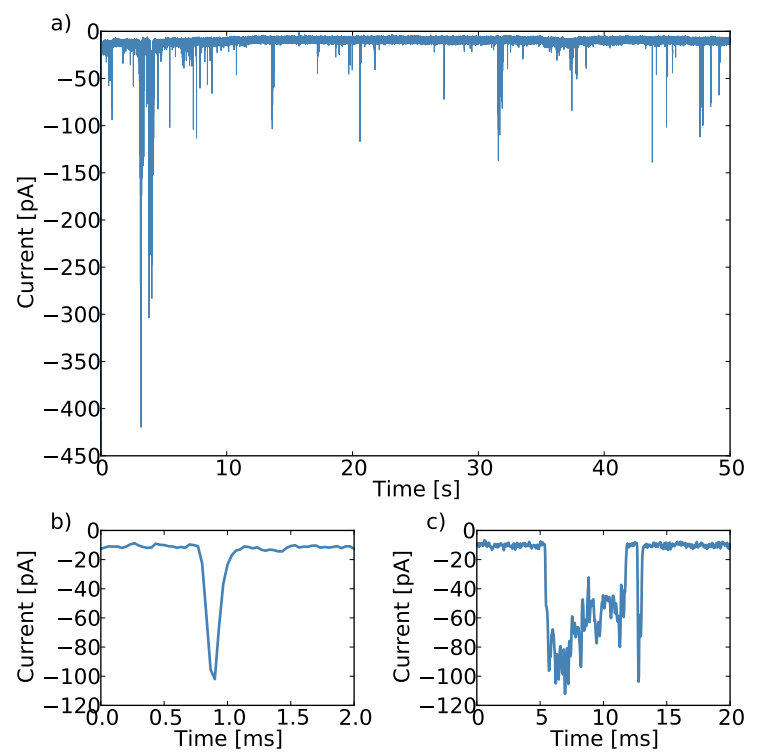

Figure 5: Chronoamperometric data of $9 \mathrm{pM}$ catalase in a $100 \mathrm{mM}$ hydrogen peroxide solution at an applied potential of $-1.0 \mathrm{~V}$ versus SCE. a) depicts the entire measurement, b) and c) amplify current feature observed at about $5.478 \mathrm{~s}$ and $47.61 \mathrm{~s}$, respectively. Please note that the time axes in b) and c) have been offset for clarity.

$$
E+S \leftrightharpoons E S \longrightarrow+\text { ne } E+P^{\prime}
$$

where $P^{\prime}$ is different from $P$. If the electron transfer step is extremely fast this scheme would not be inconsistent with the magnitude of the observed spikes. In reaction (10) the formation of $E S$ from $E$ and $S$ could proceed exactly as in bulk solution where the Michaelis-Menten kinetics operates but the reaction of $E S$ would occur via a fast electron transfer from the electrode rather than proceeding as in the Michaelis-Menten mechanism to form $P$. Thus the enzyme, in this model, acts as in bulk solution but the proximity of the electrode allows a faster alternative route for the reaction of ES namely via electron transfer instead of the usual decomposition seen in bulk solution to form $P$.

\section{Conclusions}

We provide a comprehensive theoretical and experimental analysis of the detection of individual enzymes via the nano-impact approach. In the light of recent literature, we critically evaluate the feasibility of the method to the case of impacting enzymes in a solution comprising an excess amount of substrate, if individual enzymes are to be detected electrochemically via their product. Herein, we note the huge differences in the diffusion coefficients of the enzyme and it's product: The speed of the product diffusion exceeds the enzyme's by two orders of magnitude, which results in the enzymes being almost stationary on the time scale of the product diffusion. Since in a one-dimensional system most product reaches the electrode after a certain time, the average current resulting from the product does hence not depend on the enzyme position obstructing the detection of individual enzymes or agglomerates thereof. The enzymes distance to the electrode is solely found reflected in the current noise as lower distances lead to a greater variance, this is however only one of multiple sources of noise and can therefore not directly be exploited for the measurement. Presented experiments observe current spikes, a direct comparison between theory and experimental data though reveals that these spikes cannot unambiguously be attributed to individual enzyme impacts, if the detection is via reaction of the enzyme reaction products at the electrode. An 
alternative explanation is that of 'direct' electrochemistry in which charge is transferred from an electrode to the substrate bound in the enzyme. The magnitudes of the nano-impact spikes seen are not inconsistent with such an interpretation.

\section{Acknowledgement}

The research leading to these results has received partial funding from the European Research Council under the European Union's Seventh Framework Programme (FP/2007-2013), ERC Grand Agreement Number 320403. Lior Sepunaru was supported by the European Commission under the Marie Curie Programme, Grand Number 629038. The contents reflect only the author's views and not the views of the European Commission. The table of context artwork and the illustration in Figure 2 comprise a protein rendered on the basis of data that was obtained from the Protein Data Bank in Europe (PDBe, http://www.ebi.ac.uk/pdbe/).

\section{Notes}

*We elaborate on the topic of fluctuating enzyme activity below in the Supporting Information 'Temporal evolution of the enzyme activity' $25 ; 26 ; 50$, where we discuss fluctuations arising from the enzyme being exposed to changing substrate concentrations as a result of its Brownian motion.

${ }^{\dagger}$ The disproportionation of hydrogen peroxide during the catalytic reaction is thought to proceed via two main reactions. The hydrogen peroxide is first reduced to water forming oxoferryl porphyrin cation radical $\left(\mathrm{Por}^{+}\right)$and, due to the high redox potential of the heme, hydrogen peroxide is oxidized, while the heme is reduced to its +3 state. The two consecutive reactions are summarised below: ${ }^{51}$

$$
\mathrm{H}_{2} \mathrm{O}_{2}+\operatorname{PorFe}(\mathrm{III}) \longrightarrow \mathrm{H}_{2} \mathrm{O}+\mathrm{Por}^{+} \mathrm{OFe}(\mathrm{IV})
$$

In the second step, the oxygen product is formed:

$$
\mathrm{H}_{2} \mathrm{O}_{2}+\mathrm{Por}^{+} \mathrm{OFe}(\mathrm{IV}) \longrightarrow \mathrm{H}_{2} \mathrm{O}+\mathrm{PorFe}(\mathrm{III})+\mathrm{O}_{2}
$$

${ }^{\ddagger}$ It is noted that $k_{\text {cat }}$ is defined as the maximum rate of substrate consumption and not the rate of product generation.

$\S$ The enzyme diffusion coefficient was estimated on the basis of the enzyme radius $r_{e}$ via Stokes-Einstein equation:

$$
D_{e}=\frac{k_{B} T}{6 \pi \eta r_{e}}
$$

where $k_{B}$ features its usual significance, $T=298 \mathrm{~K}$ is the temperature, and $\eta=10^{-3} \mathrm{~N} \mathrm{~s} \mathrm{~m}^{-2}$ is the viscosity of water at $25^{\circ} \mathrm{C}$. It is noted that $\eta$ herein depicts the viscosity and not the electrode overpotential.

\section{References}

[1] Pumera, M. ACS Nano 2014, 8(8), 7555-7558.

[2] Cheng, W.; Compton, R. G. TrAC Trend. Anal. Chem. 2014, 58, 79-89.

[3] Rees, N. V. Electrochem. Comm. 2014, 43, 83-86.

[4] Zhou, Y.-G.; Rees, N. V.; Compton, R. G. Angew. Chem. Int. Ed. 2011, 50(18), 4219-4221.

[5] Cheng, W.; Zhou, X.-F.; Compton, R. G. Angew. Chem. Int. Ed. 2013, 52(49), 12980-12982.

[6] Tschulik, K.; Haddou, B.; Omanović, D.; Rees, N. V.; Compton, R. G. Nano Res. 2013, 6(11), 836-841.

[7] Xiao, X.; Fan, F.-R. F.; Zhou, J.; Bard, A. J. J. Am. Chem. Soc. 2008, 130(49), 16669-16677.

[8] Cheng, W.; Compton, R. G. Angew. Chem. Int. Ed. 2014, 53(50), 13928-13930. 
[9] Lebègue, E.; Anderson, C. M.; Dick, J. E.; Webb, L. J.; Bard, A. J. Langmuir 2015, 31(42), 1173411739 .

[10] Sepunaru, L.; Tschulik, K.; Batchelor-McAuley, C.; Gavish, R.; Compton, R. G. Biomater. Sci. 2015, 3(6), 816-820.

[11] Lee, J. Y.; Kim, B.-K.; Kang, M.; Park, J. H. Scientific Reports 2016, 6.

[12] Dick, J. E.; Hilterbrand, A. T.; Boika, A.; Upton, J. W.; Bard, A. J. P. Natl. Acad. Sci. USA 2015, 112(17), 5303-5308.

[13] Sepunaru, L.; Plowman, B. J.; Sokolov, S. V.; Young, N. P.; Compton, R. G. Chem. Sci. 2016, 7(6), $3892-3899$.

[14] Toh, H. S.; Compton, R. G. Chem. Sci. 2015, 6(8), 5053-5058.

[15] Cheng, W.; Compton, R. G. Angew. Chem. Int. Ed. 2016, 55(7), 2545-2549.

[16] Hoeben, F. J. M.; Meijer, F. S.; Dekker, C.; Albracht, S. P. J.; Heering, H. A.; Lemay, S. G. ACS Nano 2008, 2(12), 2497-2504.

[17] English, B. P.; Min, W.; van Oijen, A. M.; Lee, K. T.; Luo, G.; Sun, H.; Cherayil, B. J.; Kou, S. C.; Xie, X. S. Nature Chem. Bio. 2006, 2(2), 87-94.

[18] Flomenbom, O.; Velonia, K.; Loos, D.; Masuo, S.; Cotlet, M.; Engelborghs, Y.; Hofkens, J.; Rowan, A. E.; Nolte, R. J. M.; Van der Auweraer, M.; de Schryver, F. C.; Klafter, J. P. Natl. Acad. Sci. USA 2005, 102(7), 2368-2372.

[19] Bard, A. J. ACS Nano 2008, 2(12), 2437-2440.

[20] Sekretaryova, A. N.; Vagin, M. Y.; Turner, A. P. F.; Eriksson, M. J. Am. Chem. Soc. 2016, 138(8), 2504-2507.

[21] Han, L.; Wang, W.; Nsabimana, J.; Yan, J.-W.; Ren, B.; Zhan, D. Faraday Discuss. 2016, in press.

[22] Lee, S.-K.; George, S. D.; Antholine, W. E.; Hedman, B.; Hodgson, K. O.; Solomon, E. I. J. Am. Chem. Soc. 2002, 124(21), 6180-6193.

[23] Pankratov, D.; Sotres, J.; Barrantes, A.; Arnebrant, T.; Shleev, S. Langmuir 2014, 30(10), 29432951.

[24] Ferapontova, E. E.; Grigorenko, V. G.; Egorov, A. M.; Börchers, T.; Ruzgas, T.; Gorton, L. J.Electroanal. Chem. 2001, 509(1), 19-26.

[25] Michaelis, L.; Menten, M. L. Biochem. Z. 1913, 49, 333-369.

[26] Johnson, K. A.; Goody, R. S. Biochem. 2011, 50, 8264-8269.

[27] Sim, E.; Sim, R. Eur. J. Biochem. 1979, 97(1), 119-126.

[28] Switala, J.; Loewen, P. C. Arch. Biochem. Biophys. 2002, 401(2), 145-154.

[29] Kern, D. M. H. J. Am. Chem. Soc. 1954, 76(16), 4208-4214.

[30] Ju, L.; Ho, C. Biotechnol. Bioeng. 1985, 27(10), 1495-1499.

[31] Bobbert, P. A.; Wind, M. M.; Vlieger, J. Physica A 1987, 141, 58-72. 
[32] Streeter, I.; Compton, R. G. J.Phys. Chem. C 2007, 111, 18049-18054.

[33] Fließbach, T. Statistische Physik, Lehrbuch zur Theoretischen Physik IV, 3. Auflage; Spektrum Akademischer Verlag, 1999.

[34] Eloul, S.; Kätelhön, E.; Batchelor-McAuley, C.; Tschulik, K.; Compton, R. G. J. Phys. Chem. C 2015, 119, 14400-14410.

[35] Eloul, S.; Kätelhön, E.; Batchelor-McAuley, C.; Tschulik, K.; Compton, R. G. J. Electroanal. Chem. 2015, 755, 136-142.

[36] Batchelor-McAuley, C.; Kätelhön, E.; Barnes, E. O.; Compton, R. G.; Laborda, E.; Molina, A. ChemistryOpen 2015, 4(3), 224-260.

[37] Brenner, H. Chem. Eng. Sci. 1961, 16(3-4), 242-251.

[38] Bevan, M. A.; Prieve, D. C. J. Chem. Phys. 2000, 113(3), 1228-1236.

[39] Kätelhön, E.; Compton, R. G. Chem. Sci. 2014, 5(12), 4592-4598.

[40] Kätelhön, E.; Sokolov, S. V.; Compton, R. G. Sensor. Actuat. B-Chem. 2016, 234, 420-425.

[41] Kätelhön, E.; Tanner, E. E.; Batchelor-McAuley, C.; Compton, R. G. Electrochim. Acta 2016, 199 , $297-304$.

[42] Kätelhön, E.; Feng, A.; Cheng, W.; Eloul, S.; Batchelor-McAuley, C.; Compton, R. G. J. Phys. Chem. C 2016, 120(30), 17029-17034.

[43] Bronstein, I. N.; Semendjajew, K. A.; Musiol, G.; Mühlig, H. Taschenbuch der Mathematik; Verlag Harry Deutsch, 2001.

[44] Taylor, J.; Huang, Q. CRC handbook of electrical filters; CRC Press, 1997.

[45] Matsumoto, M.; Nishimura, T. ACM T. Model. Comp. Sim. 1998, 8(1), 3-30.

[46] Batchelor-McAuley, C.; Ellison, J.; Tschulik, K.; Hurst, P. L.; Boldt, R.; Compton, R. G. Analyst 2015, $140(15), 5048-5054$.

[47] Yeager, E. Electrochim. Acta 1984, 29(11), 1527-1537.

[48] Nissim, R.; Compton, R. G. Phys. Chem. Chem. Phys. 2013, 15(28), 11918.

[49] Sepunaru, L.; Laborda, E.; Compton, R. G. Chem. Eur. J. 2016, 22(17), 5904-5908.

[50] Compton, R. G.; Banks, C. E. Understanding voltammetry; Imperial College Press, 2011.

[51] Alfonso-Prieto, M.; Biarnés, X.; Vidossich, P.; Rovira, C. Aug 2009, 131(33), 11751-11761. 